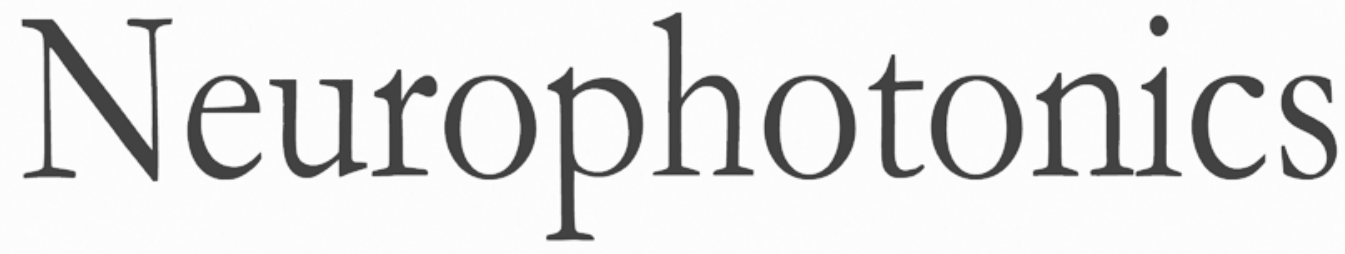

\title{
Multisite microLED optrode array for neural interfacing
}

\author{
Niall McAlinden \\ Yunzhou Cheng \\ Robert Scharf \\ Enyuan Xie \\ Erdan $\mathrm{Gu}$ \\ Christopher F. Reiche \\ Rohit Sharma \\ Prashant Tathireddy \\ Martin D. Dawson \\ Loren Rieth \\ Steve Blair \\ Keith Mathieson
}




\title{
Multisite microLED optrode array for neural interfacing
}

\author{
Niall McAlinden, ${ }^{\mathrm{a}, \star, \dagger}$ Yunzhou Cheng, ${ }^{\mathrm{a}, \dagger}$ Robert Scharf, ${ }^{\mathrm{b}}$ Enyuan Xie, ${ }^{a}$ Erdan Gu, ${ }^{\mathrm{a}}$ Christopher F. Reiche, \\ Rohit Sharma, ${ }^{b}$ Prashant Tathireddy, ${ }^{\mathrm{b}}$ Martin D. Dawson, ${ }^{\mathrm{a}}$ Loren Rieth, ${ }^{\mathrm{c}}$ Steve Blair, ${ }^{\mathrm{b}}$ and Keith Mathieson ${ }^{\mathrm{a}}$ \\ aUniversity of Strathclyde, SUPA, Institute of Photonics, Department of Physics, Glasgow, United Kingdom \\ bUniversity of Utah, Department of Electrical and Computer Engineering, Salt Lake City, Utah, United States \\ ${ }^{c}$ Feinstein Institute for Medical Research, Manhasset, New York, United States
}

\begin{abstract}
We present an electrically addressable optrode array capable of delivering light to 181 sites in the brain, each providing sufficient light to optogenetically excite thousands of neurons in vivo, developed with the aim to allow behavioral studies in large mammals. The device is a glass microneedle array directly integrated with a custom fabricated microLED device, which delivers light to 100 needle tips and 81 interstitial surface sites, giving two-level optogenetic excitation of neurons in vivo. Light delivery and thermal properties are evaluated, with the device capable of peak irradiances $>80 \mathrm{~mW} / \mathrm{mm}^{2}$ per needle site. The device consists of an array of $18180 \mu \mathrm{m} \times 80 \mu \mathrm{m}^{2}$ microLEDs, fabricated on a 150- $\mu \mathrm{m}$-thick GaN-on-sapphire wafer, coupled to a glass needle array on a $150-\mu \mathrm{m}$ thick backplane. A pinhole layer is patterned on the sapphire side of the microLED array to reduce stray light. Future designs are explored through optical and thermal modeling and benchmarked against the current device. (๑) The Authors. Published by SPIE under a Creative Commons Attribution 4.0 Unported License. Distribution or reproduction of this work in whole or in part requires full attribution of the original publication, including its DOI. [DOI: 10.1117/1.NPh.6.3.035010]

Keywords: optogenetics; microLED; optical modeling; waveguide; neurotechnology.

Paper 19019RR received Mar. 12, 2019; accepted for publication Aug. 5, 2019; published online Aug. 28, 2019.
\end{abstract}

\section{Introduction}

In a little over ten years, optogenetics has established itself as a key technique for modulating neuronal activity. ${ }^{1}$ It has been used in groundbreaking experiments in the mouse brain, allowing neuroscientists to decode neuronal circuits ${ }^{2,3}$ and examine the behavioral consequences of activity in certain neuronal populations. ${ }^{4}$ More recently, optogenetic techniques have also been applied in the nonhuman primate, ${ }^{5,6}$ the system closest to the human brain, which plays an important role in our understanding of neural computation, cognition, and behavior. Validation of optogenetic techniques in the nonhuman primate may also allow for a better understanding of neural disorders, which could lead to new clinical applications. The rapid growth of the field has been driven by significant developments in both genetically encoded light-sensitive ion channels and methods for transmitting light into neuronal tissue with the required intensity, spatial resolution, and temporal characteristics. In particular, the use of high spatial resolution stimulation has applications for combined behavioral and physiological studies, which seek to correlate neural activity with specific perceptual and cognitive functions. ${ }^{7}$ To date, most light delivery methods have focused on small rodent experiments, and there is now a recognized need for devices specifically designed for nonhuman primate studies. $^{8}$ The device detailed here, shown in Fig. 1, can provide 181 individually addressable illumination sites ( 81 for surface illumination and 100 for illumination in intracortical layers). Each site can provide a range of intensities allowing either high-resolution stimulation or larger volume stimulation $\left(0.046 \mathrm{~mm}^{3}\right)$ by controlling the drive current. Several sites can also be operated simultaneously to extend the illumination

*Address all correspondence to Niall McAlinden, E-mail: niall.mcalinden@ strath.ac.uk

†Joint first author contribution. volume further $\left(\sim 0.55 \mathrm{~mm}^{3}\right)$ or allow complex illumination patterns. This ability to produce spatiotemporal patterns of light at two layers within the cortex promises to help studies aimed at linking the neural activity of small groups of neurons to specific cognitive functions.

The main difficulty in coupling light into the brain is scattering in brain tissue. This problem is exacerbated as most opsins require blue or blue-green light to function, which experiences high tissue scattering. There are two solutions to this problem: an implantable device that delivers light directly to the region of interest, or a variety of methods allowing for excitation of opsins with infrared (IR) light at which scattering is reduced. ${ }^{9-11}$ Although using IR light is an attractive proposition, the lack of available opsins at this wavelength or the need for complex optical systems, if using two-photon methods, limits applicability in freely behaving mammals. ${ }^{9}$

To deliver light deep into brain tissue, in localized volumes, several different devices for optogenetics have been designed. The simplest and most common approach is using a fiber optic cannula, ${ }^{12}$ which allows for a single large area optogenetic excitation site. Light is normally coupled to the cannula using a fiber optic, where the resultant tethering can restrict animal movement, affecting behavior. However, devices are becoming available that couple an LED directly to the cannula and allow for more natural animal movement. ${ }^{13}$ This type of device is suitable for nonhuman primate studies where only a single excitation source is required. Coupling of a single LED into multiple fibers has also been tested, giving multisite illumination, but without individual control of illumination sites. ${ }^{14}$

Another device used for optogenetics consists of a probe structure with integrated microLEDs. ${ }^{15-20}$ These devices allow for tens to hundreds of individually controllable excitation sites, with each exciting a small volume of tissue $\left(<0.01 \mathrm{~mm}^{3}\right)$. They are electrically addressed, meaning there is potential for making a scalable, wireless device. ${ }^{19,20}$ Integrated microLED probes of 

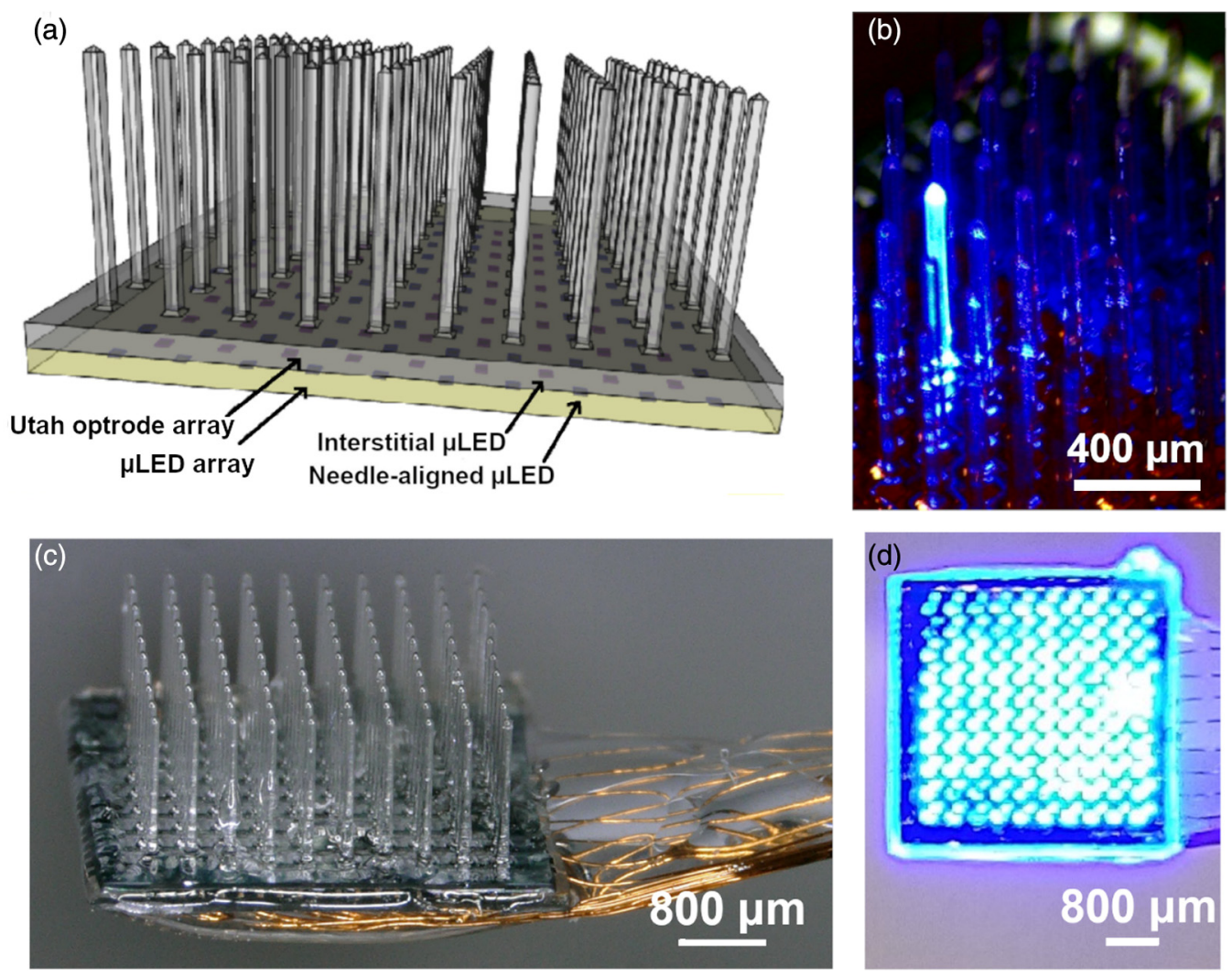

Fig. 1 (a) Schematic of the integrated device with the glass Utah optrode array (UOA) bonded to a microLED array. Illumination is from the microLED, through the sapphire (which is bonded to the glass UOA) and delivered to tissue either by the glass needles or through interstitial sites. A pinhole layer (not shown) is patterned onto the sapphire substrate of the microLED array before bonding to reduce optical crosstalk. (b) Experimental device showing illumination of a single microLED delivering high-intensity light to the needle tip. (c) Example of a fully-integrated device (not used here) prepared for in vivo experiments where polymer coated wire bonds permit independent control of the microLEDs. (d) All 100 optrode sites simultaneously illuminated.

this type provide excellent spatial and temporal control of light patterns, but require advanced thin film coatings to protect the implanted active devices - an ongoing research area.

Waveguide devices provide an alternative solution that allow for some of the advantages of both the simple fiber optical cannula approach and the microLED probe approach. The light source is external to the brain and is coupled via a waveguide probe to the target brain region. ${ }^{21,22}$ This allows for excellent spatial and temporal control of light patterns and each excitation point can illuminate a relatively large volume of tissue ( $\sim 0.1 \mathrm{~mm}^{3}$, suitable for primate behavioral studies). However, a significant drawback is the complicated and bulky optics required to couple light into the device. ${ }^{21}$ The complicated optics, with a large fiber optic bundle, restrict in vivo experiments to either head fixed or tethered arrangements.

In 2014, Pisanello et al. ${ }^{23}$ developed tapered optical fibers that allow some spatial control over the light delivered to the brain. This approach exploits the modal demultiplexing properties of tapered optical fibers, which also allowed illumination of very large volumes. ${ }^{24}$ Their elegant solution still requires significant external optics and light sources that may limit behavioral studies.

As optogenetic studies progress, there is a clear need to develop new light delivery devices, specifically designed for nonhuman primate experiments. Lee et al..$^{25}$ have developed a penetrating multifunctional needle device, specifically designed for primate studies, which can deliver light into intracortical brain structures with the required intensity to illuminate volumes of $\sim 0.1 \mathrm{~mm}^{3}$ while simultaneously recording from neurons. However, the device requires the animal to be head fixed to maintain optical alignment. Similarly, a device based on the form factor of the Utah electrode array (the Utah optrode array-UOA) has also been developed with primate studies in mind. ${ }^{26}$ This device allowed the coupling of light into intracortical brain regions with excellent spatial control, but still relied on sophisticated optical delivery systems that required tethered approaches, such as a spatial light modulator, a digital light projector, or a Holobundle system. ${ }^{27}$

In this paper, we propose a significant improvement to the UOA device by coupling the glass needle array to a custom made microLED array. This produces a compact, lightweight device that is ideally suited to primate behavioral studies. Direct integration of the light sources on to the UOA implant removes the need for complex external optics. Furthermore, since the light sources are electrically driven, they can be powered by small battery packs of the type that have been used with the Utah electrode array ${ }^{28}$ (the electrical equivalent of the optical device presented here). ${ }^{29}$ Similar devices have been developed by Kwon et al. ${ }^{24,30}$ and Schwaerzle et al., ${ }^{25,31}$ which also aim to replace external optics by directly integrating LEDs on to the penetrating needles. Their use of LEDs with low optical power densities and large standoff distances between the LED and 
wave guiding structures resulted in low optical power densities $\left(<2 \mathrm{~mW} / \mathrm{mm}^{2}\right)$ at each illumination site and hence small illumination volumes. This limits both the number of neurons that can be addressed and the optogenetic constructs used, which in many cases require higher irradiance values. These are issues, we address in this work as well as extending the number of excitation points to 181 .

Coupling LED light into a multimode waveguide such as the UOA is challenging and, without complex lens systems, low coupling efficiencies $(\sim 5 \%)$ are to be expected. ${ }^{32}$ We comprehensively model the system to maximize the efficiency of coupling and develop a prototype for testing of the optical throughput and beam profiles. Thermal measurements are also made to ensure the device adheres to the strict thermal limits of an in vivo implant. These thermal limits place restrictions on the devices operation depending on the current used and number of microLEDs illuminated.

\section{Methods}

\subsection{Device Fabrication}

The fabricated device is a $10 \times 10$ array of glass needles, each measuring $1.5 \mathrm{~mm}$ in length and spaced at $400-\mu \mathrm{m}$ pitch (Fig. 1). The needles each have a square base of side $75 \mu \mathrm{m}$ that tapers to a submicron tip. The fabrication process flow of the UOA device is detailed fully in Scharf et al. ${ }^{33}$ and summarized in Fig. S1 in the Supplementary Material. Briefly, the tips were formed using a Disco DAD3220 dicing saw with a bevel blade. The angle of the bevel defines the taper angle. The needle shanks were then defined by column dicing; deep kerfs were made in between the pyramid tips to create rectangular pillars. A wet etch step (using 49\% HF: $37 \% \mathrm{HCl}, 9: 1$ solution) is used to thin the needles. The wafer was then annealed at $725^{\circ} \mathrm{C}$ for $2 \mathrm{~h}$ to reduce the surface roughness of the shanks, which greatly reduces the scattering along the needle length. ${ }^{33}$

A custom-designed and microfabricated microLED chip is bonded directly to the backplane of the glass needle array. The microLED array is fabricated from 3-in. GaN-on-sapphire wafers (Xiamen Powerway Advanced Material) with a 400- $\mu \mathrm{m}$ thick sapphire substrate and a 5- $\mu \mathrm{m}$ epitaxial GaN layer stack. ${ }^{34}$ Full fabrication details are included in Fig. S2 in the Supplementary Material and associated text. The microLED geometry can be considered as two interleaved arrays - a $10 \times$ 10 array aligned to the glass needle shanks, and a $9 \times 9$ array positioned at interstitial sites between needles. The individual microLEDs are electrically connected using a matrix-addressing scheme (see Fig. S3 in the Supplementary Material). The glass needles guide light to intracortical brain regions, while the interstitial sites couple light to surface volumes of the brain.

\subsection{Optical Properties}

In order to understand the optical coupling to the needle, the resultant light spread in tissue, and to optimize device design, we modeled the system using the ray-tracing software, Zemax 12 , in nonsequential mode. A model was developed that represented the fabricated device [Figs. 2(a)-2(c)]. It consisted of a GaN layer patterned into individual pixels, each with a quantum well (QW) structure where light generation occurs. To model the complete device, the microLED structure, with sapphire backplane, was coupled to the needle array. The model used the peak emission wavelength of our GaN microLEDs, $450 \mathrm{~nm}$.
Refractive indices and absorption constants were taken from the standard Zemax libraries and the glass was modeled as BOROFLOAT $^{\circledR}$ 33. In each simulation run, a number of photons were launched from the LED active region such that $>10,000$ photons crossed a modeled detector surface (thus ensuring an error of $<1 \%$ from counting statistics). They were launched from a random location in the QW structure in a random direction. When they reach an interface, they are reflected/refracted [Fig. 2(c)]. In subsequent analysis, an additional absorbing layer (100\% absorption), with an aperture above each microLED, was also included to help localize light delivery to the tissue [Figs. 2(e)-2(g)]. We refer to this as a pinhole layer throughout. Brain tissue was modeled using a Henyey-Greenstein scattering model with a scattering length of $10 \mathrm{~mm}^{-1}$, absorption length of $0.07 \mathrm{~mm}^{-1}$, and anisotropy of $0.88 .^{35}$

The optical power of the microLEDs was measured using an optical power meter with a known collection angular aperture. Given that our devices have an electrical to light power conversion efficiency of $10 \%$ to $15 \%$, typical for LEDs of this wavelength, ${ }^{36}$ we can calculate the total light generated by the QW structure for input to our models. For example, a square microLED of $80 \mu \mathrm{m}$ side has an internal structure typically producing $80-\mathrm{mW}$ optical power. ${ }^{37,38}$ The efficiency figures quoted in this paper are light output as a percentage of total light generated by the microLED. This should not be confused with a coupling efficiency, quoted as a percentage of LED forward light output.

\subsection{Thermal Properties}

Brain temperature is a carefully regulated physiological variable. Small increases in temperature $\left(>1^{\circ} \mathrm{C}\right)$ can have profound physiological effects. ${ }^{39}$ Since the microLED device is in physical contact with the implanted needles, thermal properties need to be understood, quantified, and optimized. We measured the thermal characteristics of the complete system through thermal imaging (FLIR SC7000 series thermal camera). A calibration curve was first obtained as the temperature dependent emissivity of BOROFLOAT ${ }^{\circledR} 33$ glass is required. This was obtained by attaching a thermocouple to the device and heating to $80^{\circ} \mathrm{C}$. Thermal images were correlated with the thermocouple temperature as it cooled from $80^{\circ} \mathrm{C}$. The microLEDs were then driven at various currents, pulse widths, and frequencies, and temperatures recorded from the thermal imager. This method of measuring the thermal performance of our devices gave accurate readings of the temperature rise from $0.2^{\circ} \mathrm{C}$ to $60^{\circ} \mathrm{C}$. Below this range, the measurement was dominated by detector noise and above this range the IR detector saturates. This limited our thermal measurements to device currents from 20 to $100 \mathrm{~mA}$. Temperatures were recorded at the tip of our needle device directly above the active microLED.

Modeling (COMSOL Multiphysics) was completed to help understand the measured thermal properties and to give an indication as to how the device will behave in brain tissue. We verified the model by comparing outputs with the experimental results in air and extended this to make predictions of temperature rises in brain tissue by altering the specific heat and thermal conductivity, taken as $3650 \mathrm{~J} \mathrm{~kg}^{-1}{ }^{\circ} \mathrm{C}^{-1}$ and $0.5 \mathrm{~W} \mathrm{~m}^{-1}{ }^{\circ} \mathrm{C}^{-1}$ for brain tissue, respectively. ${ }^{40}$

\section{Results}

In order to integrate the UOA with the microLED device, the sapphire substrate is coupled to the glass directly. While this 


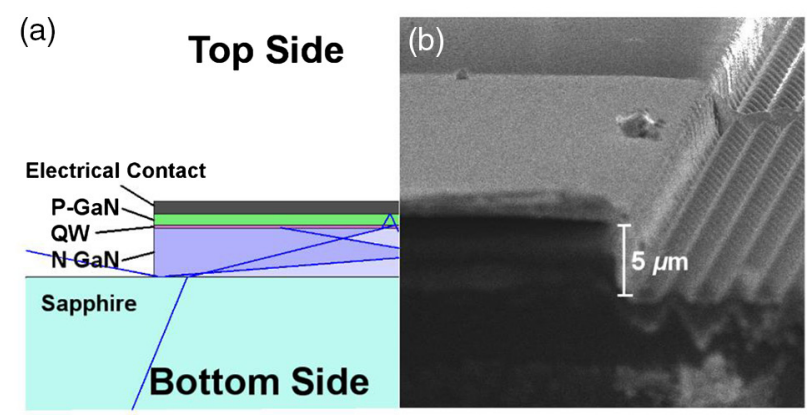

(d)
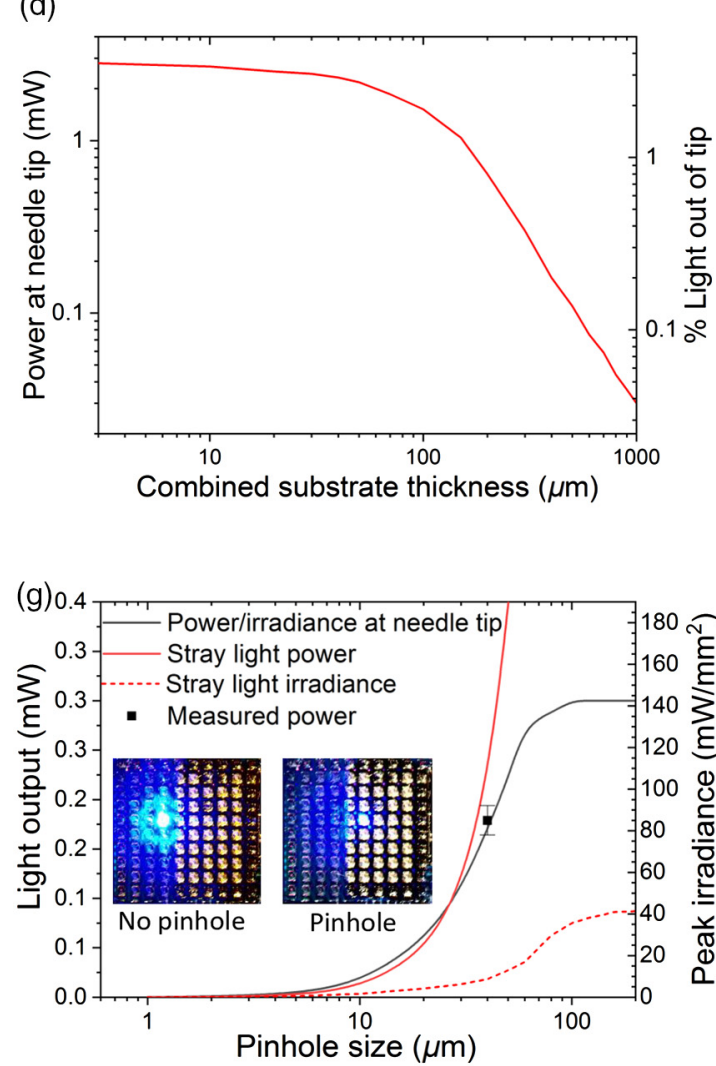
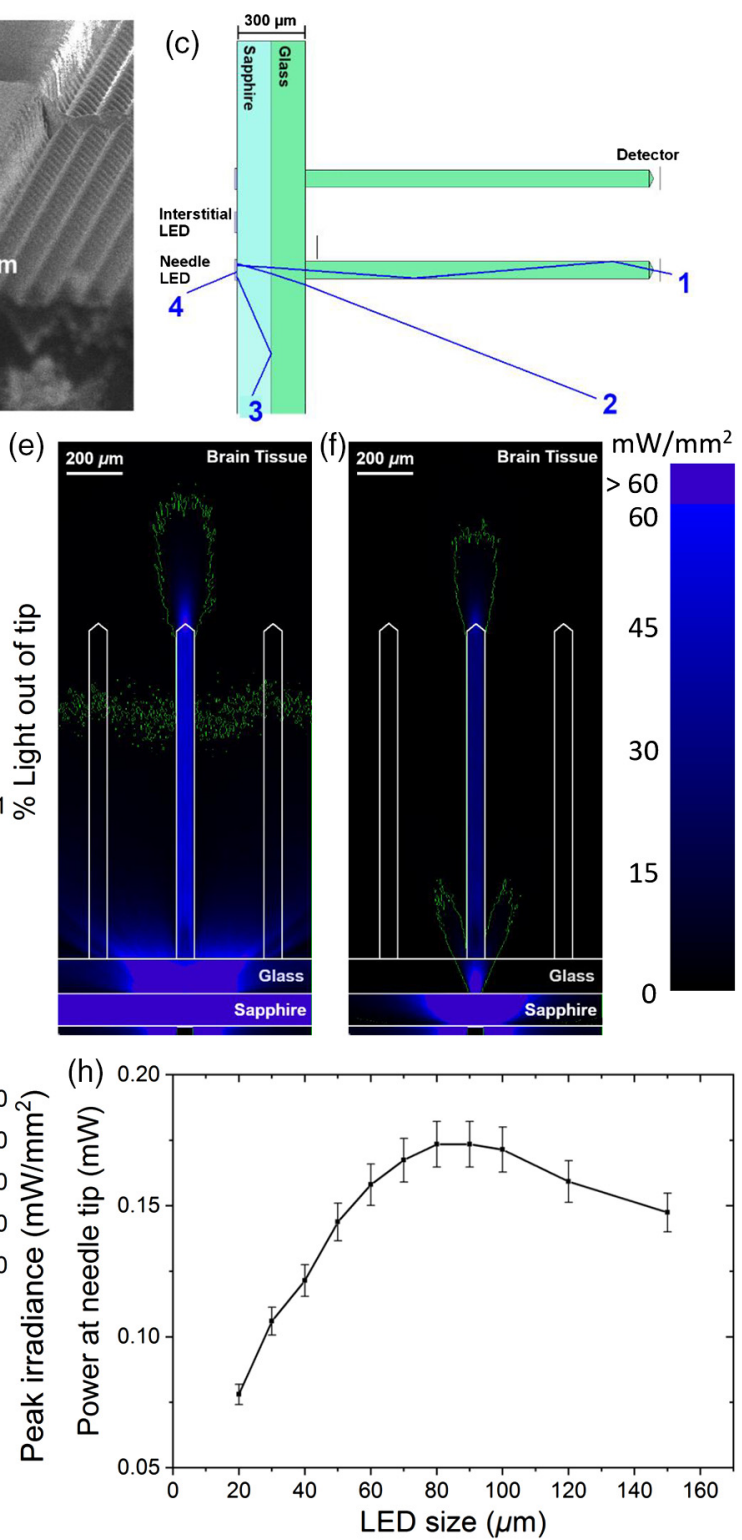

Fig. 2 (a) Schematic of the modeled microLED mesa-structure, showing the $\mathrm{p}$ - and $\mathrm{n}$-GaN layers and the GaN light-emitting QW structure on a sapphire backplane. (b) SEM image of a cleaved LED mesa-structure. Left: GaN mesa-structure. Right: Sapphire substrate (pyramidal microstructure for strain relief in QWs). (c) Cross section schematic of the integrated device. Light from the LED mesa was modeled propagating through the sapphire and glass backplane and into the glass needles; four possibilities for ray propagation are shown: (1) light coupled into the needle, (2) stray light, (3) trapped light, and (4) back emission. (d) Modeled optical power output at needle tip as a function of substrate thickness. The minimum combined substrate thickness, for mechanically reliable devices, was $300 \mu \mathrm{m}$ (150 $\mu \mathrm{m}$ sapphire, $150 \mu \mathrm{m}$ glass). (e) Modeled emission profile of an LED (20-mA drive current)—outcoupled stray light means selective excitation is not possible. (f) Incorporating an absorbing layer, with a pinhole, minimizes stray light delivery to tissue and allows selective excitation at the needle tip. In both (e) and (f), the green line indicates the $1-\mathrm{mW} / \mathrm{mm}^{2}$ contour, taken here as the irradiance threshold for ChR2. (g) Optimization of pinhole size and light delivery. Here, we consider the case for maximum light output (100-mA drive current) to understand the highest irradiance possible and the level of stray light. Insets show images of a fabricated device with a single needle-aligned microLED illuminated for two cases: with a 40- $\mu \mathrm{m}$ pinhole structure and without. (h) MicroLED size also plays a role in total light output at needle tips.

is mechanically preferable, it compromises the efficiency of the light coupling into the needles, which is dominated by the microLED to needle-base distance. Figure 2(d) shows the modeled optical power at the needle tip (as a percentage of light generated in the microLED mesa-structure) as the combined substrate thickness is varied. Below a substrate thickness of
$40 \mu \mathrm{m}$, the coupling efficiency is dominated by the acceptance numerical aperture of the glass needle. Our prototype device had a combined substrate thickness of $300 \mu \mathrm{m}$ (150 $\mu \mathrm{m}$ sapphire and $150 \mu \mathrm{m}$ glass).

In order to put our device into context, we have compared our approach to commercially available alternative LEDs (CREE, 
EZ1950-p LED, square of $1.95 \mathrm{~mm}$ side). This device obviously does not allow patterns of light, and its use with the optrode array would result in illuminating multiple needles simultaneously. Modeling this configuration shows that the peak irradiance at a current of $700 \mathrm{~mA}$ is $2.4 \mathrm{~mW} / \mathrm{mm}^{2}$ at central needle sites $\left(1.7 \mathrm{~mW} / \mathrm{mm}^{2}\right.$ at central interstitial sites). This falls away toward the edge of the device, with needles at the edge of the device having a maximum irradiance of $1.9 \mathrm{~mW} / \mathrm{mm}^{2}$. This demonstrates that although these commercial LEDs have very high power outputs, their large area means that the power density is small, when compared to the microLED approach. The microLEDs offer a high power density, advantageous when coupling light into the small apertures of the needle arrays, as well as offering patterned activation-crucial for this application.

Due to the Lambertian emission profile from LEDs, ${ }^{41}$ there will be significant stray light, even with microLEDs. Figure 2(e) shows this by showing the irradiance cross-section as light from a microLED is coupled out of the device into brain tissue. This unwanted light needs to be blocked so that it does not excite optogenetic proteins away from the intended activation region. We have implemented a method to block this stray light using an absorbing layer with pinholes (aligned to needle shanks or interstitial sites) patterned on the interface between the microLED device and needle array. Figure 2(f) shows the cross section of the emission from a needle with a $40-\mu \mathrm{m}$ pinhole-the effect of the pinhole is clearly visible. To remove the remaining stray light (at the base of the needles), it may be necessary to further coat that area with an absorbing layer. In Fig. 2(g), square optical pinholes of various dimensions ranging from 1 to $200 \mu \mathrm{m}$ were modeled. Photons were detected on two modeled detector surfaces, one at the base of the needle and one at the tip of the needle. For pinhole sizes less than $10 \mu \mathrm{m}$, light was only coupled into the needle. However, this size limits the peak irradiance at the needle tip to less than $10 \mathrm{~mW} / \mathrm{mm}^{2}$. As the pinhole size was increased, more stray light coupled out around the base of the needle. To allow for sufficient light at the tip of the needle, a pinhole size of $40 \mu \mathrm{m}$ was deemed optimal. The measured and modeled optical properties of our prototype device are summarized in Table 1, where close agreement can be seen. The optical performance of the interstitial sites is more straightforward, similar in nature and discussed fully in Fig. S4 in the Supplementary Material. MicroLED size also plays a role in optical coupling efficiency, where typical geometries are square microLEDs with sides of 5 to $200 \mu \mathrm{m}$ or circular

Table 1 Modeled and measured performance of the integrated device. Here, light emission is through the $150-\mu \mathrm{m}$ thick sapphire substrate of the microLED array and then passes through the backplane (also $150 \mu \mathrm{m}$ ) of the UOA before coupling into the glass needles. In one case, a metal absorber layer with pinhole apertures is incorporated between the sapphire and glass. Most of the light trapping occurs within the GaN mesa-structure of the microLED.

\begin{tabular}{lcccc} 
& $\begin{array}{c}\text { Light } \\
\text { output } \\
\text { at tip- } \\
\text { model (\%) }\end{array}$ & $\begin{array}{c}\text { Stray } \\
\text { light- } \\
\text { model } \\
(\%)\end{array}$ & $\begin{array}{c}\text { Trapped/ } \\
\text { absorbed } \\
\text { light- } \\
\text { model (\%) }\end{array}$ & $\begin{array}{c}\text { Light output } \\
\text { @ 100 mA- } \\
\text { measured } \\
(\%)\end{array}$ \\
\hline No pinhole & $\begin{array}{c}0.4 \\
(330 \mu \mathrm{W})\end{array}$ & 16.1 & 79.3 & $\begin{array}{c}0.45 \\
(360 \mu \mathrm{W})\end{array}$ \\
$40 \mu \mathrm{m}$ pinhole & $\begin{array}{c}0.2 \\
(170 \mu \mathrm{W})\end{array}$ & 0.3 & 95.3 & $\begin{array}{c}0.22 \\
(179 \mu \mathrm{W})\end{array}$ \\
\hline
\end{tabular}

equivalents. ${ }^{37,38}$ Figure 2(h) shows how the optical power at the needle tip varies with microLED size, with an optimum size of 70 to $100 \mu \mathrm{m}$ observed.

Guided by the modeling results, we fabricated microLED arrays with square pixels of $80 \mu \mathrm{m}$ side and a Ti:Au absorber layer $(20 \mathrm{~nm}: 30 \mathrm{~nm})$ with $40-\mu \mathrm{m}$ pinholes for needle-aligned microLEDs and 60- $\mu \mathrm{m}$ pinholes for the interstitial sites. A fabrication yield of $>99 \%$ per array was achieved, with a typical optical power-current-voltage (L-I-V) relationship shown in Fig. 3(a). The device was inserted into a fluorescent agarose solution to map the light distribution by imaging the fluorescent photons with a CMOS camera [Fig. 3(b)]. The measured beam width and depth dependence, for a microLED current of $20 \mathrm{~mA}$, matches well with the modeling giving us the confidence to extend our optical modeling to brain tissue. Figure 3(c) shows how the peak irradiance in brain tissue varies with device current. Since the needle tips produce a near-field focus for the delivered light, it is important to consider the tissue volume above a certain optical stimulation threshold (taken as $1 \mathrm{~mW} /$ $\mathrm{mm}^{2}$ here). This is shown in Fig. 3(d) as a function of microLED current. When operating at a current of $100 \mathrm{~mA}$, the device is capable of illuminating a brain tissue volume of $0.046 \mathrm{~mm}^{3}$ with $>1 \mathrm{~mW} / \mathrm{mm}^{2}$ irradiance (taking the measured optical output of the needle and using our optical model to determine the volume of tissue illuminated). If we define a minimum threshold volume for optogenetic stimulation as $0.001 \mathrm{~mm}^{3}$ (a volume of neural tissue containing $\sim 10$ neurons), then to illuminate this volume at $>1 \mathrm{~mW} / \mathrm{mm}^{2}$ irradiance requires a microLED current of just $3 \mathrm{~mA}$.

In Fig. 3(e), we show the modeled emission profile from our prototype device at various microLED currents and examine how the optical fields interact. Above a current of $50 \mathrm{~mA}$, the optical excitation volumes from neighboring needles begin to overlap.

The device was thermally tested to assess operating limits. Figures 4(a)-4(c) show the measured thermal performance versus duty cycle of the device at 20,50 , and $100 \mathrm{~mA}$ in air. The duty cycle is defined as the ratio of pulse width to interpulse period. Due to the significant thermal load between the microLEDs and the inserted needles, we find that the average temperature rise, for a fixed duty cycle, is constant, i.e., $10 \mathrm{~ms}$ pulses at $10 \mathrm{~Hz}$ give the same temperature rise as $100 \mathrm{~ms}$ pulses at $1 \mathrm{~Hz}$. To understand how these measurements, taken in air, correlate with thermal performance in brain tissue, we modeled both cases. Figures 4(a)-4(c) also show a clear match between thermal modeling and measurements for air and the resultant thermal behavior in brain tissue. All 181 LEDs can be operated simultaneously; however, thermal limits constrain the current and duty cycle parameters. Figure 4(d) shows this duty cycle limit for various microLED currents as a function of simultaneously illuminated sites.

\section{Discussion}

There is a clear need for an optogenetic device that can deliver both structured light and large volume illumination to nonsurface brain structures in larger mammals. ${ }^{8}$ The microLED-UOA neurophotonic technology introduced here is capable of providing sufficient light to excite thousands of neurons per needle site in nonhuman primates or other large mammals. The integrated nature of this electrically addressable device has the potential to enable wireless operation and so open the technology to wider neuroscience applications. In parallel with this, the fabrication 


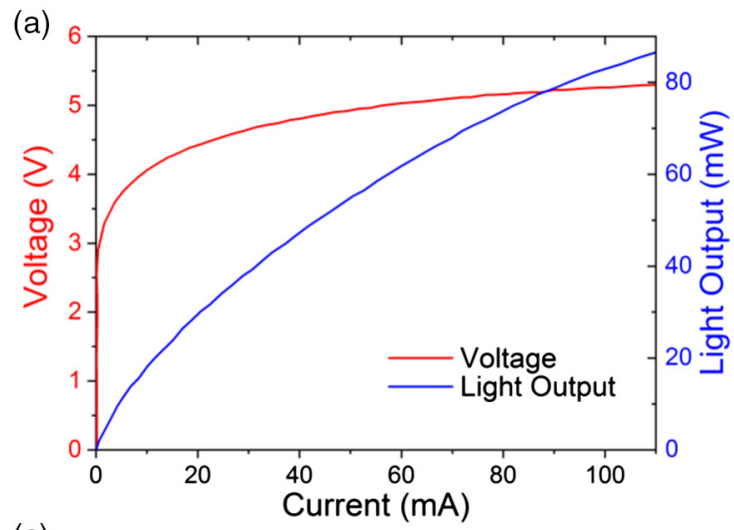

(c)

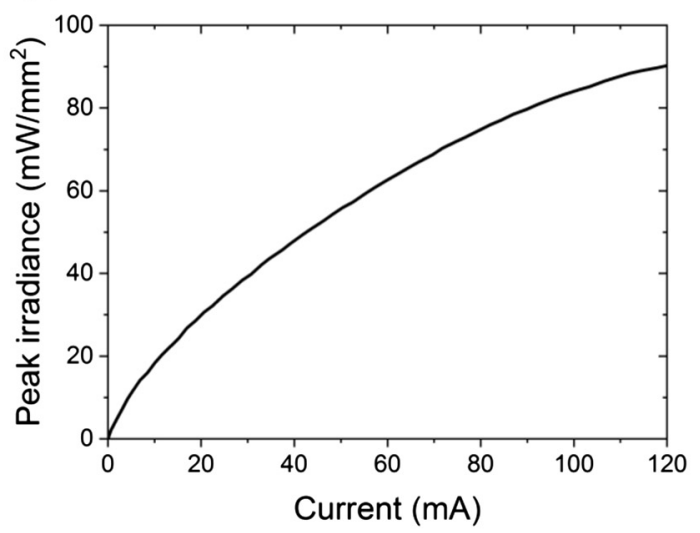

(e)

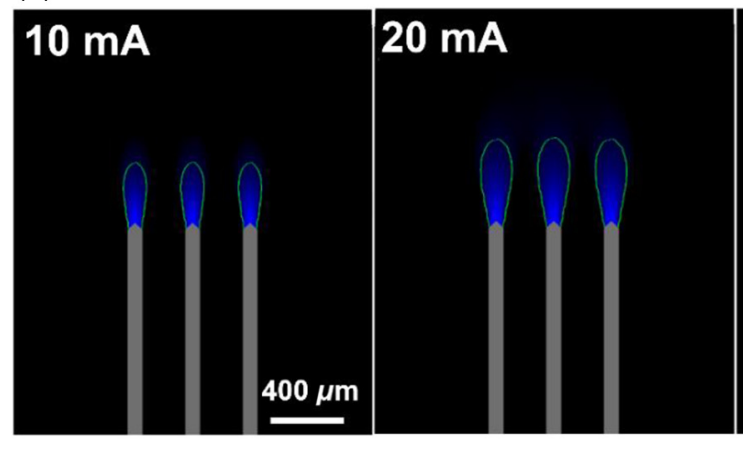

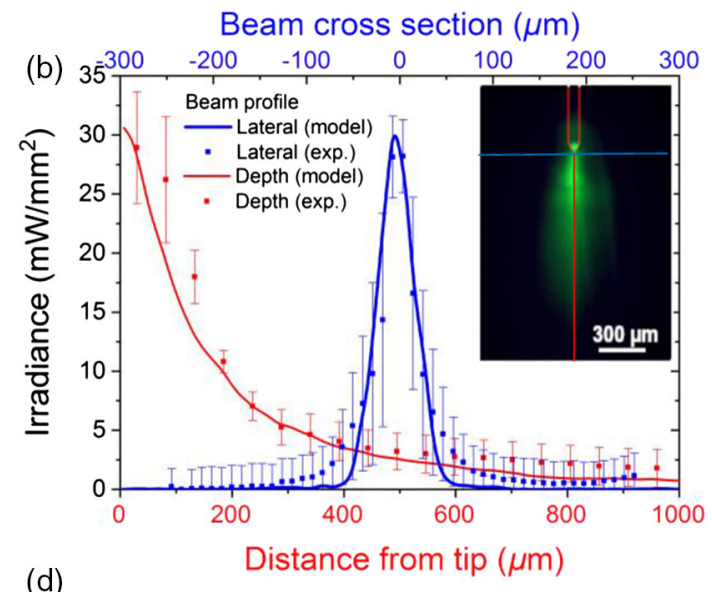

(d)

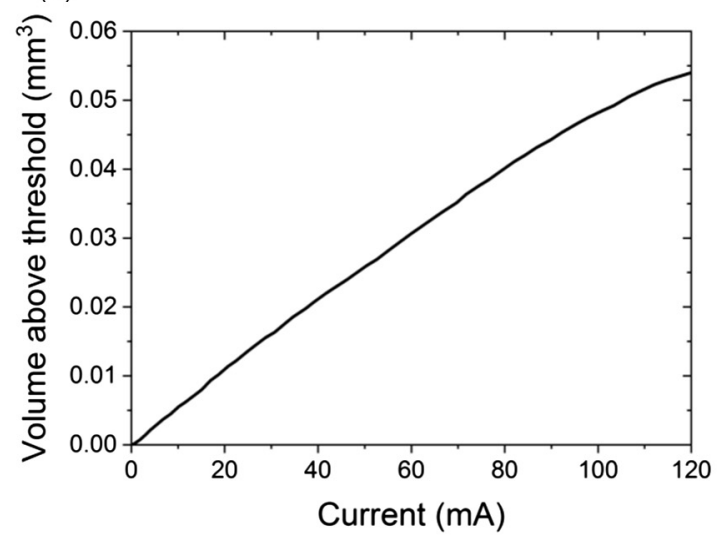

Fig. 3 (a) Current-voltage and current-light output characteristics of a typical microLED in our arrays. The microLEDs have sub-nA leakage currents and a turn-on voltage of $\sim 3 \mathrm{~V}$, indicating high quality diodes. A typical microLED produces $80 \mathrm{~mW}$ of optical power at $100 \mathrm{~mA}$ current. (b) Experimental and modeled characterization of light output from a UOA needle in fluorescein solution. Red lines (dashed, experimental; solid, modeled) show the depth dependence of the irradiance for $20 \mathrm{~mA}$ current. Blue lines (dashed, experimental; solid, modeled) the cross-section dependence $100 \mu \mathrm{m}$ from the needle tip. Inset: CMOS camera image of fluorescein excitation from the UOA. Blue and red lines indicate the position of the cross sections detailed in the main plot. (c) Peak irradiance at the tip of the needle as a function of microLED current. (d) Volume above a threshold irradiance of $1 \mathrm{~mW} / \mathrm{mm}^{2}$ as a function of microLED drive current for a device with a 40- $\mu \mathrm{m}$ pinhole. (e) Cross section of the modeled optical output in brain tissue from nine $(3 \times 3$ array) simultaneously illuminated optrodes at various microLED currents. The green contour highlights the $1-\mathrm{mW} / \mathrm{mm}^{2}$ threshold (modeled device includes the pinhole structure, $\left.40 \mu \mathrm{m}^{2}\right)$.
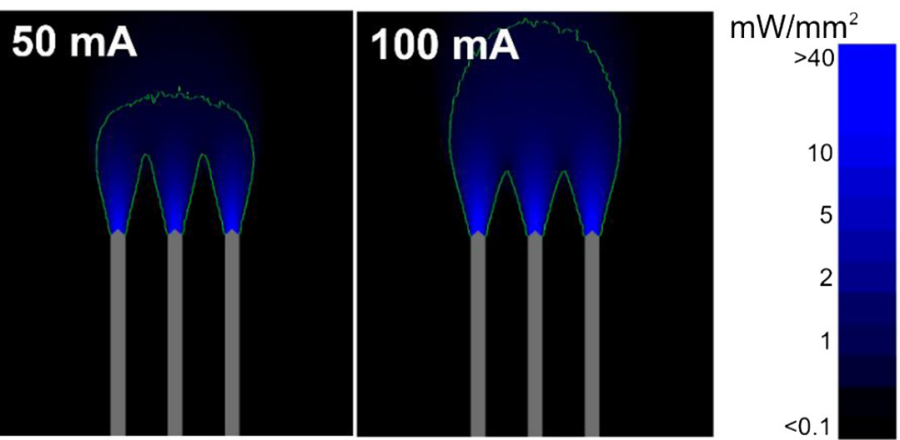

process is highly scalable and should now allow for the rapid production and distribution of the devices. The device is based on the standard format of the Utah electrode array, so that it can be handled and inserted in vivo using the pneumatic method commonly employed, ${ }^{42}$ which ensures optrode implantation while minimizing injury to delicate neural tissue. ${ }^{27}$
Comprehensive electrical, optical, and thermal testing of the microLED-UOA device was performed, alongside extensive modeling. Optical cross-talk between the glass needles can reach significant levels if strategies to block stray light are not adopted. The simplest of such was a metallic absorbing layer with small apertures, aligned with the microLEDs, and 


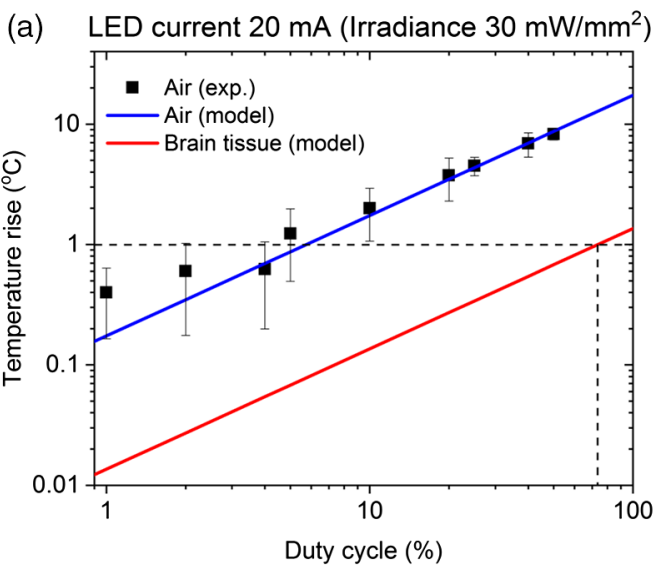

(c) LED current $100 \mathrm{~mA}$ (Irradiance $80 \mathrm{~mW} / \mathrm{mm}^{2}$ )

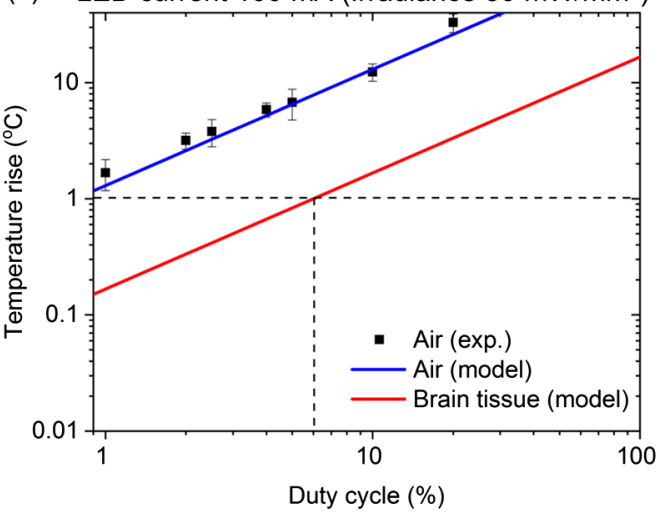

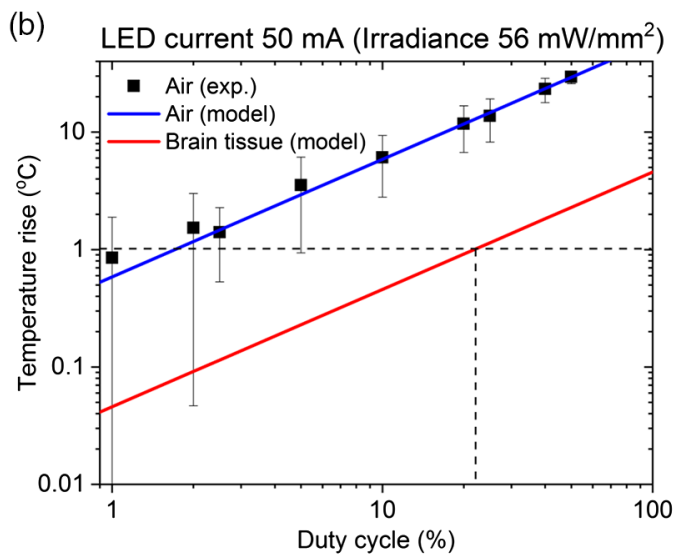

(d)

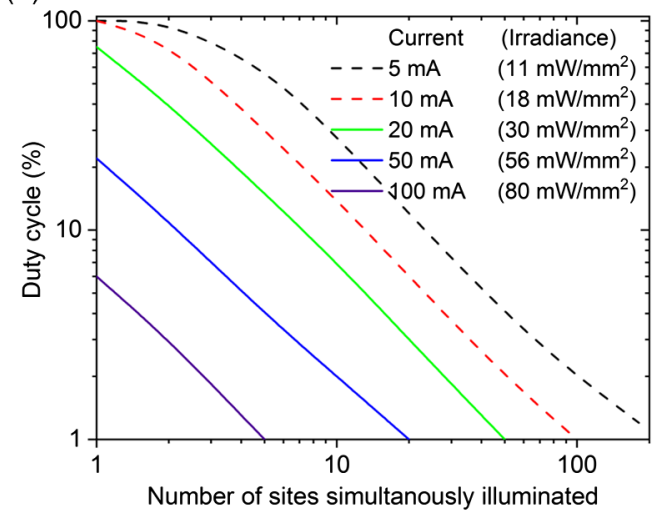

Fig. 4 (a)-(c) The temperature rise for 20, 50, and $100 \mathrm{~mA}$ microLED drive currents measured and modeled in air at the tip of the needle, with the models extended to show temperature effects in brain tissue. The duty cycle limit for a $1^{\circ} \mathrm{C}$ increase is highlighted with a dashed line. (d) The duty cycle limit is plotted against the total number of LEDs illuminated for various currents. The solid lines represent COMSOL modeled temperature rises at the tip of the needle in brain tissue (verified by measurements in air), while the dashed lines (submeasurement limit) represent modeled temperature rises in brain tissue extrapolated using the known efficiency of the microLEDs.

deposited on the sapphire side of the microLED chip. The final prototype device could emit $170 \mu \mathrm{W}$ of light at $450 \mathrm{~nm}$ wavelength from the tip of each needle, when driven at $100 \mathrm{~mA}$. This gave a peak irradiance of $80 \mathrm{~mW} / \mathrm{mm}^{2}$ at the tip of the needle. Modeling indicates that this is sufficient to optogenetically excite a volume of tissue of $0.046 \mathrm{~mm}^{3}$, containing $\sim 5500$ neurons (approximating neuronal density to $1.2 \times$ $10^{5}$ neurons $/ \mathrm{mm}^{343}$ ). It should be noted that not all of these neurons will express the optogenetic constructs and be lightsensitive, since the efficiency of opsin expression can vary and depends on a number of factors. ${ }^{6}$ Thermal measurements and modeling allow us to place a duty cycle limit on the operation of the device to ensure that neural tissue does not exceed a $1{ }^{\circ} \mathrm{C}$ temperature rise. This limit is $75 \%$ for a microLED operating at $20 \mathrm{~mA}\left(30 \mathrm{~mW} / \mathrm{mm}^{2}\right), 22 \%$ at $50 \mathrm{~mA}\left(56 \mathrm{~mW} / \mathrm{mm}^{2}\right)$, and $6 \%$ at $100 \mathrm{~mA}\left(80 \mathrm{~mW} / \mathrm{mm}^{2}\right)$. The matrix addressing scheme allows for spatiotemporal patterns of LEDs to be illuminated. This is restricted by thermal constraints, which can be considered in terms of the current supplied to the microLED, the duty cycle of the optical stimulation, and the number of LEDs active at that time. For example, illuminating five LEDs at $20 \mathrm{~mA}$ places a $15 \%$ duty cycle limit if the temperature in brain tissue at the tip is to be kept below $1{ }^{\circ} \mathrm{C}$. Illuminating 50 LEDs simultaneously, at this current, places a duty cycle limit of $1 \%$ on the device operation. For ease of reference, the thermal limits are detailed in Table S1 in the Supplementary Material for various pulse widths, repetition rates, and numbers of simultaneously active microLEDs and span typical optogenetic operating ranges.

Modeling also allowed the optical design to be optimized. The pinhole structure used to block stray light in our prototype device is not ideal. It cannot block all stray light and still allow for enough light at the needle tip. An alternative option would be to replace the glass backplane of the needle array with a silicon backplane. Through-silicon-vias can be opened in this Si backplane, allowing light to be efficiently coupled into needle and interstitial sites and forming an optical interposer. ${ }^{33}$ This silicon-interposer design has the added advantage that it can be thinned to tens of microns (using advanced silicon processing) while still allowing for a mechanically stable device with high fabrication yield. This thinning of the integrated device brings the microLED and needle aperture closer and yields the biggest gain in efficiency. By switching to a top emitting LED, we can minimize the microLED-needle stand-off distance and design a device with an effective backplane of only $90 \mu \mathrm{m}$ (optical adhesive and Si interposer thickness). These alternative device configurations are modeled in Fig. S5 in the Supplementary Material, with Table S2 in the Supplementary 
Material detailing the optical efficiency gains. These approaches have the potential to increase the optical efficiency of our device threefold (from $0.2 \%$ to $0.6 \%$ ), corresponding to a peak irradiance of $184 \mathrm{~mW} / \mathrm{mm}^{2}$ and increasing the maximum illumination volume to $>0.1 \mathrm{~mm}^{3}$ (per needle site), while also ensuring there is no optical cross talk. Furthermore, by increasing the optical efficiency, there is an associated power reduction for a given irradiance, which in turn reduces the operating temperature. An increase in optical efficiency from $0.2 \%$ to $0.6 \%$ would reduce the power required for each microLED from 0.5 to $0.113 \mathrm{~W}$ for a peak irradiance of $80 \mathrm{~mW} / \mathrm{mm}^{2}$. This would mean that $50 \mathrm{~ms}$ pulses, at a maximum repetition rate of $10 \mathrm{~Hz}$ (up from $1 \mathrm{~Hz}$ ), would be safely within thermal limits.

\section{Disclosures}

Loren Rieth and Prashant Tathireddy were partial employees of Blackrock Microsystems until April 2017 during which part of this work was conducted. No conflicts of interest, financial or otherwise, are declared by the other authors.

\section{Acknowledgments}

This work was supported by the NIH BRAIN Initiative Program, through Grant No. U01 NS099702. KM was supported by the Royal Academy of Engineering under the Chair in Emerging Technologies scheme.

\section{References}

1. K. Deisseroth, "Optogenetics: 10 years of microbial opsins in neuroscience," Nat. Neurosci. 18, 1213-1225 (2015).

2. B. J. Hunnicutt et al., "A comprehensive thalamocortical projection map at the mesoscopic level," Nat. Neurosci. 17, 1276-1285 (2014).

3. G. J. Kress et al., "Convergent cortical innervation of striatal projection neurons," Nat. Neurosci. 16, 665-667 (2013).

4. A. I. Domingos et al., "Leptin regulates the reward value of nutrient," Nat. Neurosci. 14, 1562-1568 (2011).

5. X. Han, "Optogenetics in the nonhuman primate," Prog. Brain Res. 196, $215-233$ (2012)

6. I. Diester et al., "An optogenetic toolbox designed for primates," Nat. Neurosci. 14, 387-397 (2011).

7. M. R. Cohen and W. T. Newsome, "What electrical microstimulation has revealed about the neural basis of cognition," Curr. Opin. Neurobiol. 14(2), 169-177 (2004).

8. A. Galvan et al., "Nonhuman primate optogenetics: recent advances and future directions," J. Neurosci. 37(45), 10894-10903 (2017).

9. R. Prakash et al., "Two-photon optogenetic toolbox for fast inhibition, excitation and bistable modulation," Nat. Methods 9, 1171-1179 (2012).

10. S. Hososhima et al., "Near-infrared (NIR) up-conversion optogenetics," Sci. Rep. 5, 16533 (2015).

11. K. G. Chernov et al., "Near-infrared fluorescent proteins, biosensors, and optogenetic tools engineered from phytochromes," Chem. Rev. 117(9), 6423-6446 (2017).

12. F. Zhang et al., "Optogenetic interrogation of neural circuits: technology for probing mammalian brain structures," Nat. Protocols 5, 439-456 (2010).

13. G. Gagnon-Turcotte et al., "A wireless optogenetic headstage with multichannel electrophysiological recording capability," Sensors 15(9), 22776-22797 (2015).

14. S. Jeon et al., "Multi-wavelength light emitting diode-based disposable optrode array for in vivo optogenetic modulation," J. Biophotonics 12, e201800343 (2019).

15. G. Buzsáki et al., "Tools for probing local circuits: high-density silicon probes combined with optogenetics," Neuron 86(1), 92-105 (2015).

16. N. McAlinden et al., "Optogenetic activation of neocortical neurons in vivo with a sapphire-based micro-scale LED probe," Front. Neural Circuits 9, 25 (2015).
17. R. Scharf et al., "Depth-specific optogenetic control in vivo with a scalable, high-density $\mu$ LED neural probe," Sci. Rep. 6, 28381 (2016).

18. F. Wu et al., "Monolithically integrated $\mu$ LEDs on silicon neural probes for high-resolution optogenetic studies in behaving animals," Neuron 88(6), 1136-1148 (2015).

19. T.-I. Kim et al., "Injectable, cellular-scale optoelectronics with applications for wireless optogenetics," Science 340(6129), 211-216 (2013).

20. P. Gutruf et al., "Fully implantable optoelectronic systems for batteryfree, multimodal operation in neuroscience research," Nat. Electron. 1(12), 652-660 (2018).

21. A. N. Zorzos, E. S. Boyden, and C. G. Fonstad, "Multiwaveguide implantable probe for light delivery to sets of distributed brain targets," Opt. Lett. 35(24), 4133-4135 (2010).

22. L. Hoffman et al., "High-density optrode-electrode neural probe using SixNy photonics for in vivo optogenetics," in IEEE Int. Electron Dev. Meeting (IEDM) (2015).

23. F. Pisanello et al., "Multipoint-emitting optical fibers for spatially addressable in vivo optogenetics," Neuron 82(6), 1245-1254 (2014).

24. F. Pisanello et al., "Dynamic illumination of spatially restricted or large brain volumes via a single tapered optical fiber," Nat. Neurosci. 20, 1180-1188 (2017).

25. J. Lee et al., "Transparent intracortical microprobe array for simultaneous spatiotemporal optical stimulation and multichannel electrical recording," Nat. Methods 12, 1157-1162 (2015).

26. T. V. F. Abaya et al., "A 3D glass optrode array for optical neural stimulation," Biomed. Opt. Express 3(12), 3087-3104 (2012).

27. R. W. Boutte et al., "Utah optrode array customization using stereotactic brain atlases and 3-D CAD modeling for optogenetic neocortical interrogation in small rodents and nonhuman primates," Neurophotonics 4(4), 041502 (2017).

28. X. Xie et al., "Long-term reliability of $\mathrm{Al}_{2} \mathrm{O}_{3}$ and Parylene $\mathrm{C}$ bilayer encapsulated Utah electrode array based neural interfaces for chronic implantation," J. Neural Eng. 11(2), 026016 (2014).

29. C. A. Chestek et al., "HermesC: low-power wireless neural recording system for freely moving primates," IEEE Trans. Neural Syst. Rehabil. Eng. 17(4), 330-338 (2009).

30. K. Y. Kwon et al., "Opto- $\mu$ ECoG Array: A Hybrid Neural Interface With Transparent $\mu \mathrm{ECoG}$ Electrode Array and Integrated LEDs for Optogenetics," IEEE Trans. Biomed. Circuits Syst. 7(5), 593-600 (2013).

31. M. Schwaerzle et al., "Miniaturized $3 \times 3$ optical fiber array for optogenetics with integrated $460 \mathrm{~nm}$ light sources and flexible electrical interconnection," in IEEE Int. Conf. Micro Electro Mech. Syst. (MEMS), IEEE, Estoril, Portugal, pp. 162-165 (2010).

32. G. Keiser, Optical Fiber Communications, 4th ed., McGraw-Hill Education, New York (2008).

33. R. Scharf et al., "A compact integrated device for spatially selective optogenetic neural stimulation based on the Utah optrode array," Proc. SPIE 10482, 104820M (2018).

34. J.-H. Ryouand and W. Lee, "GaN on sapphire substrates for visible light-emitting diodes," in Nitride Semiconductor Light-Emitting Diodes (LEDs), J. Huang, H.-C. Kuo, and S.-C. Shen, Eds., 2nd ed., pp. 43-78, Woodhead Publishing, Cambridge (2018).

35. J. G. Bernstein et al., "Prosthetic systems for therapeutic optical activation and silencing of genetically targeted neurons," Proc. SPIE 6854, 68540H (2008).

36. A. A. Greshnov et al., "Comparative study of quantum efficiency of blue LED with different nanostructural arrangement," Phys. Status Solidi C 4(8), 2981-2985 (2007).

37. P. Tian et al., "Size-dependent efficiency and efficiency droop of blue InGaN micro-light emitting diodes," Appl. Phys. Lett. 101(23), 231110 (2012).

38. J. J. D. McKendry et al., "Visible-light communications using a CMOScontrolled micro-light- emitting-diode array," J. Lightwave Technol. 30(1), 61-67 (2012).

39. P. Andersen and E. I. Moser, "Brain temperature and hippocampal function," Hippocampus 5(6), 491-498 (1995).

40. M. E. Maged et al., "Bio-heat transfer model of deep brain stimulationinduced temperature changes," J. Neural Eng. 3(4), 306-315 (2006).

41. C. Griffin et al., "Beam divergence measurements of InGaN/GaN micro-array light-emitting diodes using confocal microscopy," Appl. Phys. Lett. 86(4), 041111 (2005). 
42. P. J. Rousche and R. A. Normann, "A method for pneumatically inserting an array of penetrating electrodes into cortical tissue," Ann. Biomed. Eng. 20(4), 413-422 (1992).

43. C. E. Collins et al., "Neuron densities vary across and within cortical areas in primates," Proc. Natl. Acad. Sci. U. S. A. 107(36), 15927-15932 (2010).

Niall McAlinden was awarded his PhD in surface and interface optics from Trinity College Dublin in 2010. Since then, he has worked at the Institute of Photonics at the University of Strathclyde. He works on a variety of biophotonics and imaging projects. His primary focus is on the development of optoelectronic devices for optogenetics, including innovative microLED devices.

Yunzhou Cheng will receive his $\mathrm{PhD}$ in physics from the University of Strathclyde, Glasgow, United Kingdom, in August, 2019. He currently works as a research associate at the Institute of Photonics, University of Strathclyde. His work mainly focuses on the fabrication and characterization of optoelectronic semiconductor devices.

Robert Scharf received his Dipl.-Ing. degree in mechanical engineering from the University of Hannover, Germany, in 2012. He was awarded his $\mathrm{PhD}$ from the Institute of Photonics, University of Strathclyde in 2017. During his PhD, he developed high density optoelectronic devices for studying neural tissue. Since then he has worked at the University of Utah, Department of Electrical and Computer Engineering.

Enyuan Xie received his $\mathrm{PhD}$ in physics from the University of Strathclyde, Glasgow, United Kingdom, in 2013. Since then, he has been with the Institute of Photonics, University of Strathclyde, as a research associate, becoming involved in the fabrication, characterization, and application of GaN-based micro-LED arrays.

Erdan Gu has been working at the Institute of Photonics, University of Strathclyde, Glasgow since 2002. He received his PhD from the University of Aberdeen, United Kingdom, in 1992. Then, he worked as a research fellow in the Cavendish Laboratory, University of Cambridge, United Kingdom. In 1997, he joined Oxford Instruments plc, United Kingdom. As an associate director at the Institute of Photonics, he plays a leading role in a range of research projects.

Christopher F. Reiche is a research assistant professor with the Department for Electrical and Computer Engineering at the University of Utah. His research focuses on the development of innovative microdevices for sensing applications, as well as scanning force microscopy and corresponding materials research. He received his diploma in physics from the Karlsruhe Institute of Technology, Germany, in 2011, and his research doctorate in physics from the Technische Universität Dresden, Germany, in 2016.

Rohit Sharma: Biography is not available.

Prashant Tathireddy received his bachelor's degree in chemical technology from Osmania University, Hyderabad, India, in 1997, and his PhD from the Department of Chemical Engineering, University of Utah, Salt Lake City, in 2005. He is currently with Applied Biosensors, leading the development of continuous multi-analyte sensors for use in biopharmaceutical and human clinical applications.

Martin D. Dawson is a distinguished professor and director of research at the University of Strathclyde's Institute of Photonics, which he helped found almost 25 years ago. He is also, since 2012, the head of the UK's first Fraunhofer research centre: the Fraunhofer Centre for Applied Photonics. He is recognized for his wide-ranging work on lasers and compound semiconductors, including pioneering contributions to VECSELs, diamond photonics, and micro-LEDs.

Loren Rieth is an associate professor in the Institute for Bioelectronic Medicine at the Feinstein Institute for Medical Research, Manhasset, New York, and adjunct in Electrical and Biomedical Engineering Departments at the University of Utah. His research focuses on development and translation of neural interface technologies to the central and peripheral nervous system. He received his PhD from the University of Florida in 2001 and his BS degree from Johns-Hopkins in 1994 in materials science and engineering.

Steve Blair received his BS and MS degrees from Rose-Hulman Institute of Technology in 1991 and 1993, respectively, and his PhD from the University of Colorado at Boulder in 1998. Since 1998, he has been with the Electrical and Computer Engineering Department at the University of Utah in Salt Lake City. His research interests include neural interfaces, plasmonics, slow-light nonlinear optics, photonic microsystems, and microarray technology.

Keith Mathieson received his BSc and $\mathrm{PhD}$ degrees in physics from the University of Glasgow. He has been supported by fellowships from the Royal Society of Edinburgh and SU2P to develop retinal implants. He worked at Stanford University (Palanker Lab, 2009 to 2011), developing an optoelectronic retinal prosthesis and is now at the University of Strathclyde where he holds a Royal Academy of Engineering Professorial Chair in Emerging Technologies. His research interests are in neural interface technologies. 